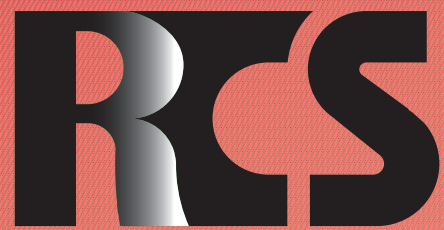

Depósito legal ppi $201502 Z U 4662$

Esta publicación científica en formato digital es continuidad de la revista impresa Depósito Legal: pp $197402 Z$ Z789

- ISSN: 1315-9518 • ISSN-E: 2477-9431

Revista de Ciencias Sociales

Universidad del Zulia. Revista de la Facultad de Ciencias Económicas y Sociales

Vol. XXVII,

No. Especial 3, 2021

Esta publicación científica en formato digital es continuidad de la revista impresa Depósito Legal: pp $197402 Z$ Z789

ISSN: $1315-9518$ 


\title{
Producción de biomasa en cultivos de maíz: Zona central de la costa de Ecuador
}

\author{
Vásconez Montúfar, Gregorio Humberto* \\ Caicedo Acosta, Luis Alberto** \\ Véliz Zamora, Diana Verónica*** \\ Sánchez Mora, Fernando David ${ }^{* * * *}$
}

\section{Resumen}

La reducción de la brecha entre el rendimiento actual y el alcanzable de maíz, representa una oportunidad para incrementar los beneficios de los productores, para el efecto como primer paso, es pertinente evaluar la producción de biomasa del maíz. En un diseño de bloques completos aleatorizados, se estudiaron tres híbridos de maíz, en los que se determinó la distribución de biomasa a nivel de tallo, hojas y mazorca, y la absorción de nutrientes a la cosecha. Se realizó análisis de varianza, prueba de Tukey y análisis de regresión, considerando la acumulación de biomasa en función del tiempo. Los híbridos evaluados alcanzaron la máxima acumulación de biomasa total a los 60 días. La acumulación de biomasa vegetativa total (tallo más hoja) y biomasa reproductiva, se ajustó a una curva sigmoidea, mientras que la desacumulación de biomasa vegetativa total, mantuvo una tendencia lineal simple. El rendimiento de los híbridos modernos evaluados es mayor que el observado en el híbrido antiguo, lo que responde a una mayor absorción de nutrientes y no a la acumulación y distribución de biomasa. En conclusión, sugiere que la selección de materiales con mayor capacidad de acumulación/desacumulación podría contribuir a incrementar el rendimiento de los productores de maíz.

Palabras clave: Brecha de rendimiento; agricultores; cultivo de maíz; biomasa; Ecuador.

Maestro en Nutrición Vegetal. Ingeniero Agrónomo. Docente en la Facultad de Ciencias Pecuarias de la Universidad Técnica Estatal de Quevedo, Ecuador. E-mail: gvasconez@uteq.edu.ec (iD ORCID: https://orcid.org/0000-0003-1260-8075

** Docente en la Facultad de Ciencias Pecuarias de la Universidad Técnica Estatal de Quevedo, Ecuador. E-mail: luis.caicedo@uteq.edu.ec

*** Magíster en Ciencias Vegetales mención Producción Vegetal. Docente en la Facultad de Ciencias Pecuarias de la Universidad Técnica Estatal de Quevedo, Ecuador. E-mail: dvveliz@uteq.edu.ec

(iDORCID: https://orcid.org/0000-0003-2039-8741

***** Master em Recursos Genéticos Vegetais. Docente en la Facultad de Ingeniería Agronómica de la Universidad Técnica de Manabí, Ecuador. E-mail: fernando.sanchez@utm.edu.ec (DD ORCID: https:// orcid.org/0000-0002-9078-2290

Recibido: 2021-02-21 · Aceptado: 2021-05-10 


\title{
Biomass production in corn crops: Central zone of the Ecuadorian coast
}

\begin{abstract}
The reduction of the gap between current and achievable corn yield represents an opportunity to increase the benefits of producers. For this purpose, as a first step, it is pertinent to evaluate the biomass production of corn. In a randomized complete block design, three maize hybrids were studied, in which the biomass distribution was determined at the stem, leaf and ear level, and the absorption of nutrients at harvest. Analysis of variance, Tukey's test and regression analysis were carried out, considering the accumulation of biomass as a function of time. The hybrids evaluated reached the maximum accumulation of total biomass at 60 days. The accumulation of total vegetative biomass (stem plus leaf) and reproductive biomass, was adjusted to a sigmoid curve, while the decumulation of total vegetative biomass, maintained a simple linear trend. The performance of the modern hybrids evaluated is higher than that observed in the old hybrid, which responds to a greater absorption of nutrients and not to the accumulation and distribution of biomass. In conclusion, it suggests that the selection of materials with greater accumulation / decumulation capacity could contribute to increasing the yield of corn producers.
\end{abstract}

Keywords: Performance gap; farmers; corn crop; biomass; Ecuador.

\section{Introducción}

El maíz, junto con el arroz y la soya, es un cultivo estratégico para satisfacer la creciente demanda de alimentos y de material lignocelulósico requerido por la industria de los biocombustibles (Melendez, et al., 2021), debido al aumento de la población que para el año 2050 se estima supere los 9.000 millones de habitantes (Ray, et al., 2013; Walker, 2016). Al respecto, Peña y López (2020) manifiestan que:

En la constante búsqueda de otras alternativas para frenar el calentamiento global que ayude a reducir las emisiones de $\mathrm{CO}_{2}$, aparecieron los biocombustibles a partir de los residuos lignocelulósicos, y entre los cultivos energéticos están el maíz, la caña de azúcar, el sorgo o soya; siendo éstos una fuente de energía alternativa cuyo uso contribuyen a la utilización de la biomasa, para obtener productos de alto valor agregado. (p.402)

Sin embargo, la producción de biomasa $\mathrm{y}$ el rendimiento actual se encuentra por debajo del nivel alcanzable por los cultivos, en muchos de los agroecosistemas del mundo, así en Ecuador la productividad de las provincias maiceras (Los Ríos, Guayas y Manabí) no supera las 5,5 $\mathrm{t} \mathrm{ha}^{-1}$ (Monteros y Salvador, 2014), lo cual es inferior a las $10 \mathrm{t} \mathrm{ha}^{-1} \mathrm{de}$ grano, que con facilidad pueden alcanzar los modernos híbridos de maíz comercializados en Ecuador (Vera, et al., 2020), esto sugiere que la reducción de la brecha entre la producción de biomasa actual y la alcanzable, constituye una estrategia viable para incrementar la productividad de maíz, los ingresos y por ende, mejorar el nivel de la calidad de vida de los agricultores.

El uso eficiente de los recursos, principalmente del agua y de los nutrientes, constituyen una estrategia real para reducir la brecha entre el rendimiento actual y el rendimiento alcanzable de un determinado cultivo (Fischer, Byerlee y Edmeades, 2014), pero para ello, es necesario conocer la dinámica de acumulación y distribución de biomasa a medida que el cultivo crece y se desarrolla (fenología), puesto que existe una estrecha relación entre la biomasa acumulada y la demanda de nutrientes (Nkebiwe, et al., 
2016).

Vásquez, et al. (2018) sostienen que: "Los sistemas agrícolas tradicionales o agroecosistemas se caracterizan por estabilizar el rendimiento a largo plazo, promueven la diversidad de la dieta basada en la disponibilidad de una variedad de plantas y fuentes de nutrientes" (p.25). En ese sentido, la producción de biomasa de los cultivos es particular para cada uno de los agroecosistemas, por ser altamente sensible a la: Radiación solar, temperatura, disponibilidad de agua, fertilidad del suelo y presión de enfermedades, plagas y malezas.

Lo anterior, probablemente ha provocado en Ecuador, al igual que en muchos otros países en el mundo, que todos los años se cuente con nuevos híbridos de maíz, ya sean introducidos o desarrollados por instituciones nacionales como el Instituto Ecuatoriano de Investigaciones Agropecuarias o las Universidades (Vasco, et al., 2017), y pese a esto, la brecha entre rendimiento actual y el rendimiento alcanzable para el cultivo de maíz es aproximadamente del 32\% (explicado en párrafos anteriores).

Bajo condiciones no restrictivas de humedad en el suelo y un adecuado control de enfermedades, plagas y malezas, el factor más determinante en el rendimiento es la fertilidad del suelo. Sin embargo, para la definición de un nivel adecuado de fertilidad es necesario determinar el máximo nivel de producción de biomasa de los cultivos en un determinado agroecosistema, y a partir de ello, establecer la demanda de nutrientes del cultivo y el desarrollo de estrategias de fertilización diseñadas para maximizar el rendimiento con el menor impacto ambiental (Andrade, et al., 1996).

Es fundamental reconocer la importancia de la producción agrícola para el "desarrollo de la humanidad al igual que en el uso de los recursos naturales, con el fin de implementar la utilización de productos alternativos, su estudio, así como planeación, en aras de obtener una producción optimizada, eficiente y sustentable" (Caicedo, et al., 2020, p.309). Por lo tanto, el conocimiento de la producción y distribución de biomasa, más el requerimiento de nutrientes por unidad de biomasa de tallos, hojas y mazorcas, representa un elemento significativo en el desarrollo de planes de fertilización, orientados a mejorar la eficiencia de los nutrientes en el cultivo de maíz, sabiendo que, en la mayoría de los casos, el objetivo principal del cultivo es la producción de granos, pero en otros casos, es la obtención de forraje.

Sobre la base de los antecedentes expuestos, se plantea en el presente artículo evaluar la distribución de biomasa y la absorción de nutrientes en tres híbridos de maíz, a fin de establecer la demanda de nutrientes para este cultivo, como una estrategia que permita hacer un mejor manejo del fertilizante, en la zona central del litoral ecuatoriano.

\section{Metodología}

La investigación de tipo descriptiva, se realizó en la Finca Experimental "La María", de la Universidad Técnica Estatal de Quevedo, situada en la provincia de Los Ríos en Ecuador, dentro de las coordenadas geográficas $01^{\circ} 0^{\prime}$ de latitud Sur y $79^{\circ} 29^{\prime}$ de longitud Oeste, a 75 $\mathrm{msnm}$. La zona de vida corresponde al Bosque Húmedo Tropical, en donde se ha registrado en términos promedio, en los últimos 10 años, una temperatura mínima y máxima de 21,5 y $29,3{ }^{\circ} \mathrm{C}$, respectivamente; una humedad relativa mínima y máxima de $62 \%$ y $98 \%$, respectivamente; y una precipitación anual de $2.613 \mathrm{~mm}$, distribuida entre la época lluviosa (enero a junio) y seca (julio a diciembre) en proporciones de $93 \%$ y $7 \%$, respectivamente.

Haciendo uso de la clave para la taxonomía de suelos de los Estados Unidos (Soil Survey Staff, 2014), el suelo de la Finca Experimental La María se clasificó como un Inceptisol con características ándicas. Además, siguiendo los métodos de laboratorio aceptados por la Sociedad Ecuatoriana de la Ciencia del Suelo y descritos por DíazRomeu y Hunter (1982), se determinó que el suelo tenía una concentración de materia 
Producción de biomasa en cultivos de maiz: Zona central de la costa de Ecuador

Vásconez Montúfar, Gregorio Humberto; Caicedo Acosta, Luis Alberto;

Véliz Zamora, Diana Verónica y Sánchez Mora, Fernando David

orgánica (titulación del dicromato residual con sulfato ferroso) de $24 \mathrm{~g} \mathrm{~kg}^{-1}$; de $\mathrm{N}\left(\mathrm{NH}_{4}\right)$ y P (colorimetría, Olsen modificado) de 19 y $16 \mathrm{mg} \mathrm{kg}^{-1}$, respectivamente; de $\mathrm{K}, \mathrm{Ca}$ y $\mathrm{Mg}$ (absorción atómica, Olsen modificado) de 0,5, 10,0 y $2,5 \mathrm{cmol}_{\mathrm{c}} \mathrm{kg}^{-1}$; y de $\mathrm{S}$ (turbidimetría, fosfato de calcio monobásico), $\mathrm{Zn}, \mathrm{Cu}, \mathrm{Fe}$ y Mn (absorción atómica, Olsen modificado) de $6,5,10,144$ y $9 \mathrm{mg} \mathrm{kg}^{-1}$, respectivamente.

La siembra del maíz, se realizó con un distanciamiento entre hilera y planta de 80 y $20 \mathrm{~cm}$, respectivamente, previo a la preparación del terreno con dos pases de rastra. La fertilización, se la realizó en bandas con la adición de $\mathrm{N}, \mathrm{P}, \mathrm{K}, \mathrm{Mg}$ y $\mathrm{S}$ a razón de $20,3,15,2$ y $3 \mathrm{~g} \mathrm{~m}^{-2}$, respectivamente. El control de malezas, plagas y enfermedades se lo ejecutó de manera manual con el objeto de solo registrar el efecto de los tratamientos evaluados.

Asimismo, los híbridos de maíz, evaluados durante la época lluviosa, fueron el INIAP-H551 (antiguo), UTEQ-101 (moderno), desarrollados por el Instituto Ecuatoriano de Investigaciones Agropecuarias y la Universidad Técnica Estatal de Quevedo, respectivamente, y el DK-7508 (moderno), comercializado por la empresa privada. La siembra fue dispuesta en un diseño de bloques completos al azar, con tres tratamientos (híbridos de maíz) y siete repeticiones. Las dimensiones de cada unidad experimental fue de 4 x $12 \mathrm{~m}$, en donde las tres hileras centrales de maíz, fueron consideradas útiles con el fin de controlar el efecto borde.

La distribución de biomasa a nivel de tallos, hojas y mazorca de los híbridos UTEQ-101， INIAP-H551 y DK-7508, se valoró en términos de materia seca (MS) acumulada por unidad de superficie. Para el efecto, se cosechó las plantas existentes en 1 $\mathrm{m}^{2}$ (aproximadamente entre 5 a 6 plantas $\mathrm{m}^{-2}$ ), a los 20, 40, 60, 80, 100 y 120 días después de la emergencia. La TAB (g MS mía ${ }^{-1}$ ) fue determinada haciendo uso de la Ecuación 1 , donde $B_{2}$ es la biomasa acumulada, al momento de la medida (g MS m ${ }^{-2}$; $B_{1}$ es la biomasa acumulada, en el tiempo anterior al del momento de la medida ( $\left.\mathrm{g} \mathrm{MS} \mathrm{m}^{-2}\right) ; T_{2}$ es el periodo transcurrido al momento de la medida (días); y, $T_{1}$ es el periodo transcurrido hasta el tiempo anterior al del momento de la medida (días).

$$
\text { Ecuación 1: } \quad T A B=\frac{B_{2}-B_{1}}{T_{2}-T_{1}}
$$

Si la $T A B$, es positiva se interpreta como acumulación; y si es negativa, como desacumulación de biomasa. La absorción de nutrientes se obtuvo como producto de la MS acumulada en cada uno de los órganos de la planta de maíz al momento de la cosecha (120 días calendario después de la emergencia) y la concentración de $\mathrm{N}, \mathrm{P}, \mathrm{K}, \mathrm{Mg}$ y S a nivel de tallos, hojas y mazorcas. Órganos que previamente fueron desecados en estufa de aíre forzado a $75^{\circ} \mathrm{C}$ hasta peso constante.

Los datos obtenidos de la acumulación de biomasa ( $\mathrm{g} \mathrm{MS} \mathrm{m}^{-2}$ ), TAB ( $\mathrm{g}$ MS $\mathrm{m}^{-2}$ día $^{-}$ $\left.{ }^{1}\right)$, y la absorción total de nutrientes $\left(\mathrm{g} \mathrm{kg}^{-1}\right)$ fueron sujetos a un análisis de varianza, y la comparación de medias, se realizó a través de la prueba de Tukey a un nivel de confianza del 0,05. Además, se realizó un análisis de regresión para describir, en función del tiempo, la acumulación y desacumulación de biomasa de los órganos vegetativos (tallo y hojas), y la acumulación de biomasa a nivel de mazorca (órgano reproductivo). El nivel de ajuste del modelo se lo valoró a través del coeficiente de determinación $\left(R^{2}\right)$.

\section{Resultados y discusión}

A los 20 días luego de la emergencia (417 GDA), la acumulación de biomasa a nivel del tallo no fue significativa entre los híbridos evaluados, con un promedio aproximado de $28 \mathrm{~g} \mathrm{MS} \mathrm{m}^{-2}$. Sin embargo, se observó que la máxima acumulación de biomasa fue alcanzada en distintos momentos dependiendo del híbrido, así el UTEQ-101 e INIAP-H551, alcanzaron la máxima acumulación a nivel del tallo a los 40 días, con 685 y 612 g MS $\mathrm{m}^{-2}$, respectivamente; mientras que el DK- 
7508 , la alcanzó a los 60 días (1125 GDA) con $672 \mathrm{~g} \mathrm{MS} \mathrm{m}^{-2}$. Luego de alcanzar la máxima acumulación de biomasa, los tres híbridos evaluados, desacumularon biomasa desde el tallo de manera progresiva hasta los 100 días
(1.814 GDA), registrándose una biomasa promedio final a nivel del tallo de $153 \mathrm{~g} \mathrm{MS}$ $\mathrm{m}^{-2}$, siendo la biomasa del híbrido DK-7508, significativamente mayor con $195 \mathrm{~g} \mathrm{MS} \mathrm{m}^{-2}$ (ver Tabla 1).

Tabla 1

Distribución de biomasa en tallo, hojas y mazorca en híbridos de maíz cultivados en la zona central del litoral ecuatoriano

\begin{tabular}{|c|c|c|c|c|c|c|c|c|c|c|}
\hline \multirow[t]{2}{*}{$\begin{array}{l}\text { Organo } \\
\text { vegetal }\end{array}$} & \multicolumn{2}{|c|}{ Tiempo acumulado } & \multicolumn{2}{|c|}{$\begin{array}{c}\text { UTEQ } \\
101\end{array}$} & \multicolumn{2}{|c|}{$\begin{array}{l}\text { INIAP } \\
\text { H551 }\end{array}$} & \multicolumn{2}{|c|}{$\begin{array}{c}\text { DK } \\
7508\end{array}$} & \multirow[t]{2}{*}{$\bar{X}$} & \multirow[t]{2}{*}{ EEM } \\
\hline & $\begin{array}{c}\text { Calendario } \\
\text { (día) }\end{array}$ & $\begin{array}{l}\text { Térmico } \\
\text { (GDA) }\end{array}$ & & \multicolumn{5}{|c|}{$\mathrm{g} \mathrm{MS} \mathrm{m}^{-2}$} & & \\
\hline \multirow{6}{*}{ Tallo } & 20 & 417 & 29,44 & $a *$ & 25,94 & $\mathrm{a}$ & 29,89 & $\mathrm{a}$ & 28,42 & 1,09 \\
\hline & 40 & 764 & 685,23 & a & 612,14 & $\mathrm{a}$ & 397,70 & $\mathrm{~b}$ & 565,02 & 39,04 \\
\hline & 60 & 1.125 & 554,32 & $\mathrm{a}$ & 369,86 & $\mathrm{~b}$ & 572,16 & $\mathrm{a}$ & 498,78 & 23,34 \\
\hline & 80 & 1.467 & 375,54 & $\mathrm{a}$ & 318,61 & $\mathrm{a}$ & 436,49 & $\mathrm{a}$ & 376,88 & 20,99 \\
\hline & 100 & 1.814 & 151,14 & $\mathrm{~b}$ & 114,57 & $\mathrm{~b}$ & 195,61 & $\mathrm{a}$ & 153,78 & 10,52 \\
\hline & 120 & 2.160 & 116,07 & $a b$ & 101,38 & $\mathrm{~b}$ & 142,97 & $\mathrm{a}$ & 120,14 & 5,80 \\
\hline \multirow{6}{*}{ Hojas } & 20 & 417 & 53,25 & $a b$ & 48,13 & b & 65,59 & $\mathrm{a}$ & 55,65 & 2,57 \\
\hline & 40 & 764 & 410,08 & $\mathrm{a}$ & 420,46 & $\mathrm{a}$ & 389,54 & $\mathrm{a}$ & 406,69 & 18,82 \\
\hline & 60 & 1.125 & 360,37 & b & 276,80 & $\mathrm{c}$ & 413,59 & $\mathrm{a}$ & 350,25 & 14,24 \\
\hline & 80 & 1.467 & 380,69 & a & 197,44 & $\mathrm{a}$ & 346,24 & a & 308,12 & 32,83 \\
\hline & 100 & 1.814 & 61,93 & b & 53,14 & b & 103,91 & a & 72,99 & 5,56 \\
\hline & 120 & 2.160 & 65,17 & $\mathrm{~b}$ & 66,58 & $\mathrm{~b}$ & 94,29 & a & 75,35 & 3,73 \\
\hline \multirow{4}{*}{ Mazorca } & 60 & 1.125 & 370,31 & $\mathrm{ab}$ & 418,28 & $\mathrm{a}$ & 246,75 & $\mathrm{~b}$ & 345,11 & 24,14 \\
\hline & 80 & 1.467 & 374,65 & $\mathrm{a}$ & 442,83 & $\mathrm{a}$ & 430,54 & $\mathrm{a}$ & 416,01 & 16,55 \\
\hline & 100 & 1.814 & 613,95 & b & 722,08 & $\mathrm{a}$ & 600,88 & $\mathrm{~b}$ & 645,63 & 20,34 \\
\hline & 120 & 2.160 & 709,83 & $\mathrm{a}$ & 647,44 & $\mathrm{a}$ & 637,77 & $\mathrm{a}$ & 665,01 & 15,00 \\
\hline \multirow{6}{*}{ Total } & 20 & 417 & 82,69 & $a b$ & 74,07 & b & 95,48 & a & 84,08 & 3,56 \\
\hline & 40 & 764 & $1.095,31$ & $\mathrm{a}$ & $1.032,61$ & $\mathrm{a}$ & 787,24 & $\mathrm{a}$ & 971,72 & 54,29 \\
\hline & 60 & 1.125 & $1.285,00$ & $\mathrm{a}$ & $1.064,94$ & $\mathrm{~b}$ & $1.232,49$ & $a b$ & $1.194,14$ & 33,29 \\
\hline & 80 & 1.467 & $1.130,87$ & $\mathrm{a}$ & 958,88 & $\mathrm{a}$ & $1.213,26$ & $\mathrm{a}$ & $1.101,00$ & 47,54 \\
\hline & 100 & 1.814 & 827,01 & $\mathrm{a}$ & 889,79 & $\mathrm{a}$ & 900,40 & $\mathrm{a}$ & 872,40 & 21,81 \\
\hline & 120 & 2.160 & 891,08 & $\mathrm{a}$ & 815,40 & $\mathrm{a}$ & 875,03 & $\mathrm{a}$ & 860,51 & 17,83 \\
\hline
\end{tabular}

Nota: * promedios horizontales con letras iguales no son estadísticamente diferentes de acuerdo a la prueba de Tukey al nivel 0,05. GDA, grados días acumulados. EEM, error estándar de la media.

Fuente: Elaboración propia, 2021.

La acumulación de biomasa a nivel de las hojas, a los 20 días luego de la emergencia fue diferente entre los híbridos evaluados, observándose una acumulación en los híbridos UTEQ-101 y DK-7508 de 53 y $65 \mathrm{~g} \mathrm{MS} \mathrm{m}^{-2}$, respectivamente; lo cual, fue significativamente mayor a la acumulación observada en el INIAP-H551 con 46 g MS $\mathrm{m}^{-2}$. Además, a nivel de las hojas, se observó que la máxima acumulación de biomasa fue alcanzada en distintos momentos dependiendo del híbrido. A los 40 días, los híbridos UTEQ101 e INIAP-H551 alcanzaron la máxima acumulación a nivel de las hojas, con 410 y 
$420 \mathrm{~g} \mathrm{MS} \mathrm{m}^{-2}$, respectivamente; mientras que, el DK-7508 alcanzó la máxima acumulación a los 60 días con $413 \mathrm{~g} \mathrm{MS} \mathrm{m}^{-2}$ (ver Tabla 1).

Al igual que lo observado a nivel del tallo, luego que se alcanzó la máxima acumulación de biomasa al nivel de las hojas, se observó una desacumulación de biomasa de manera progresiva hasta los 100 días, registrándose una biomasa promedio final a nivel de las hojas de $73 \mathrm{~g} \mathrm{MS} \mathrm{m}^{-2}$, siendo la biomasa de los híbridos UTEQ-101 (62 g MS m $\mathrm{m}^{-2}$ ) e INIAP-H551 (53 $\left.\mathrm{g} \mathrm{MS} \mathrm{m}^{-2}\right)$, significativamente menor con respecto a la biomasa acumulada en las hojas en el DK7508 (104 $\mathrm{g} \mathrm{MS} \mathrm{m}^{-2}$ ) (ver Tabla 1).

La formación de mazorca, y por ende la acumulación de biomasa a nivel de mazorca, en los tres híbridos de maíz evaluados, se inició entre los 40 y 60 días luego de la emergencia de las plántulas. A los 60 días, con una biomasa promedio de $394 \mathrm{~g} \mathrm{MS} \mathrm{m}^{-2}$, no hubo diferencia entre los híbridos UTEQ-101 e INIAP-H551, observándose una biomasa significativamente superior a la alcanzada por el híbrido DK-7508 (246 g MS m²). Luego de haberse iniciado la formación de mazorca, el híbrido INIAP-H551 alcanzó la máxima acumulación de biomasa a los 100 días $(722 \mathrm{~g}$ MS $\mathrm{m}^{-2}$ ), la cual fue significativamente mayor a la observada en los híbridos UTEQ-101 (709 g MS m${ }^{-2}$ ) y DK-7508 (637 g MS m-2), que alcanzaron la máxima acumulación a los 120 días $\left(2.160^{\circ}\right.$ días) (ver Tabla 1$)$.

Los tres híbridos evaluados alcanzaron la máxima acumulación de biomasa total a los 60 días, siendo los híbridos UTEQ-101 y el DK-7508 los que registraron una biomasa significativamente mayor, que la observada en el híbrido INIAP-H551 (1.064 g MS m²), con 1.285 y $1.232 \mathrm{~g} \mathrm{MS} \mathrm{m}^{-2}$, respectivamente. Luego de que los híbridos alcanzaron la máxima acumulación de biomasa, se observó una pérdida progresiva de biomasa hasta los 100 días, llegando a una biomasa estadísticamente igual entre los tres híbridos, la cual en promedio alcanzó a $872 \mathrm{~g} \mathrm{MS} \mathrm{m}^{-2}$ (ver Tabla 1).

Al evaluar la tasa de acumulación de biomasa a nivel del tallo, definida como la acumulación de materia seca por unidad de superficie y tiempo, los tres híbridos evaluados alcanzaron la mayor tasa de acumulación de biomasa a los 40 días. No obstante, el híbrido DK-7508 tuvo la menor tasa de acumulación de biomasa con $18 \mathrm{~g} \mathrm{MS} \mathrm{m}^{-2}$ día $^{-1}$, la cual fue significativamente inferior a la alcanzada en los híbridos UTEQ-101 e INIAP-H55 que fue de 32 y $29 \mathrm{~g} \mathrm{MS} \mathrm{m}^{-2}$ día $^{-1}$, respectivamente. A los 60 días los híbridos UTEQ-101 (-6 g MS $\mathrm{m}^{-2}$ día $\left.^{-1}\right)$ e INIAP-H55 (-12 g MS m $\mathrm{m}^{-2}$ día $\left.^{-1}\right)$ estaban desacumulando biomasa desde el tallo a una tasa estadísticamente equivalente, a diferencia del híbrido DK-7508 que aún acumulaba a una tasa de $8 \mathrm{~g} \mathrm{MS} \mathrm{m}{ }^{-2}$ día $^{-1}$, en el cual se evidenció tasas negativas de acumulación de biomasa (desacumulación) a partir de los 80 días (ver Tabla 2). 
Tabla 2

Tasa de acumulada de biomasa en tallo, hojas y mazorca en híbridos de maíz cultivados en la zona central del litoral ecuatoriano

\begin{tabular}{|c|c|c|c|c|c|c|c|c|c|c|}
\hline \multirow{2}{*}{$\begin{array}{l}\text { Órgano } \\
\text { vegetal }\end{array}$} & \multicolumn{2}{|c|}{ Tiempo acumulado } & \multicolumn{2}{|c|}{$\begin{array}{c}\text { UTEQ } \\
101\end{array}$} & \multicolumn{2}{|c|}{$\begin{array}{c}\text { INIAP } \\
\text { H551 }\end{array}$} & \multicolumn{2}{|c|}{$\begin{array}{c}\text { DK } \\
7508\end{array}$} & \multirow[t]{2}{*}{$\bar{X}$} & \multirow{2}{*}{ EEM } \\
\hline & $\begin{array}{l}\text { Calendario } \\
\text { (días) }\end{array}$ & $\begin{array}{c}\text { Térmico } \\
\text { (GDA) }\end{array}$ & & & - g M & 2 día & & & & \\
\hline \multirow{6}{*}{ Tallo } & 20 & 417 & 1,47 & $a^{*}$ & 1,30 & a & 1,49 & $\mathrm{a}$ & 1,42 & 0,05 \\
\hline & 40 & 764 & 32,79 & $\mathrm{a}$ & 29,31 & $\mathrm{a}$ & 18,39 & $\mathrm{~b}$ & 26,83 & 1,96 \\
\hline & 60 & 1.125 & $-6,55$ & $\mathrm{~b}$ & $-12,11$ & $\mathrm{~b}$ & 8,72 & $\mathrm{a}$ & $-3,31$ & 2,49 \\
\hline & 80 & 1.467 & $-8,94$ & $\mathrm{~b}$ & $-2,56$ & $\mathrm{a}$ & $-6,78$ & $a b$ & $-6,09$ & 0,95 \\
\hline & 100 & 1.814 & $-11,22$ & $\mathrm{a}$ & $-10,20$ & $\mathrm{a}$ & $-12,04$ & $\mathrm{a}$ & $-11,15$ & 0,98 \\
\hline & 120 & 2.160 & $-1,75$ & $\mathrm{a}$ & $-0,66$ & a & $-2,63$ & $\mathrm{a}$ & $-1,68$ & 0,45 \\
\hline \multirow{6}{*}{ Hojas } & 20 & 417 & 2,66 & $a b$ & 2,41 & b & 3,28 & a & 2,78 & 0,13 \\
\hline & 40 & 764 & 17,84 & $\mathrm{a}$ & 18,62 & a & 16,20 & $\mathrm{a}$ & 17,55 & 0,95 \\
\hline & 60 & 1.125 & $-2,49$ & $\mathrm{a}$ & $-7,18$ & b & 1,20 & $a b$ & $-2,82$ & 1,18 \\
\hline & 80 & 1.467 & 1,02 & $\mathrm{a}$ & $-3,97$ & a & $-3,37$ & $\mathrm{a}$ & $-2,11$ & 1,38 \\
\hline & 100 & 1.814 & $-15,94$ & $\mathrm{a}$ & $-7,21$ & $\mathrm{a}$ & $-12,12$ & a & $-11,76$ & 1,61 \\
\hline & 120 & 2.160 & 0,16 & $\mathrm{a}$ & 0,67 & a & $-0,48$ & $\mathrm{a}$ & 0,12 & 0,22 \\
\hline \multirow{4}{*}{ Mazorca } & 60 & 1.125 & 18,52 & $a b$ & 20,29 & $\mathrm{a}$ & 12,34 & $\mathrm{~b}$ & 17,26 & 1,21 \\
\hline & 80 & 1.467 & 0,22 & $\mathrm{~b}$ & 1,23 & $a b$ & 9,19 & $\mathrm{a}$ & 3,55 & 1,53 \\
\hline & 100 & 1.814 & 11,96 & $\mathrm{a}$ & 13,96 & $\mathrm{a}$ & 8,52 & $\mathrm{a}$ & 11,48 & 1,25 \\
\hline & 120 & 2.160 & 4,79 & $\mathrm{a}$ & $-3,73$ & $\mathrm{~b}$ & 1,85 & $\mathrm{ab}$ & 0,97 & 1,34 \\
\hline \multirow{6}{*}{ Total } & 20 & 417 & 4,13 & $a b$ & 3,70 & b & 4,77 & a & 4,20 & 0,18 \\
\hline & 40 & 764 & 50,63 & $\mathrm{a}$ & 47,93 & a & 34,59 & $\mathrm{a}$ & 44,38 & 2,76 \\
\hline & 60 & 1.125 & 9,48 & $a b$ & 1,62 & b & 22,26 & a & 11,12 & 3,13 \\
\hline & 80 & 1.467 & $-7,71$ & $\mathrm{a}$ & $-5,30$ & $\mathrm{a}$ & $-0,96$ & $\mathrm{a}$ & $-4,66$ & 2,20 \\
\hline & 100 & 1.814 & $-15,19$ & $\mathrm{a}$ & $-3,46$ & $\mathrm{a}$ & $-15,64$ & $\mathrm{a}$ & $-11,43$ & 2,53 \\
\hline & 120 & 2.160 & 3,20 & $\mathrm{a}$ & $-3,72$ & a & $-1,27$ & a & $-0,59$ & 1,38 \\
\hline
\end{tabular}

Nota: * promedios horizontales con letras iguales no son estadísticamente diferentes de acuerdo a la prueba de Tukey al nivel 0.05. GDA, grados días acumulados. EEM, error estándar de la media.

Fuente: Elaboración propia, 2021.

Semejante a lo observado a nivel de tallo, a los 40 días los tres híbridos de maíz experimentaron la mayor tasa de acumulación de biomasa, siendo la diferencia entre ellas no significativa con un promedio de $17 \mathrm{~g} \mathrm{MS}$ $\mathrm{m}^{-2}$ día $^{-1}$. A partir de los 60 días luego de la emergencia, prácticamente lo tres híbrido registraron desacumulación de biomasa a nivel de las hojas, alcanzándose la máxima tasa de desacumulación a los 100 días, siendo la diferencia entre ellas no significativa con un promedio de -11 g MS m $\mathrm{g}^{-2}$ día $^{-1}$ (ver Tabla 2).

La formación de mazorca, cuyo inicio se registró entre los 40 y 60 días, experimenta su mayor velocidad en los tres híbridos evaluados a los 60 día luego de la emergencia, con una tasa de acumulación de biomasa del híbrido DK-7508 de $12 \mathrm{~g} \mathrm{MS} \mathrm{m}^{-2}$ día $^{-1}$, la cual fue significativamente menor a la observada en los híbridos UTEQ-101 e INIAP-H551, con tasas de acumulación de biomasa de 18 y 20 g MS $\mathrm{m}^{-2}$ día $^{-1}$, respectivamente, las mismas que no fueron estadísticamente diferentes (ver Tabla 2).

Los tres híbridos evaluados alcanzaron la máxima tasa de acumulación de biomasa total a los 40 días, cuya diferencia no fue significativa entre los híbridos, con una tasa promedio de $44 \mathrm{~g} \mathrm{MS} \mathrm{m}^{-2}$ día $^{-1}$. Luego, entre los 40 y 100 días, es evidente una disminución 
sistemática de la biomasa total en los tres híbridos, siendo mayor a los 100 días, aunque no hubo diferencias significativas entre híbridos, con un valor promedio de $-11 \mathrm{~g} \mathrm{MS}$ $\mathrm{m}^{-2}$ día $^{-1}$ (ver Tabla 2).

La acumulación de biomasa vegetativa total (tallo más hojas) a través del tiempo (calendario o térmico) se ajustó a una curva sigmoidea (ecuación de Boltzmann) (ver Figura I: a, b y c), en los híbridos UTEQ$101\left(\mathrm{R}^{2}=0.878, \mathrm{p}<0.01\right)$, INIAP-H551 $\left(\mathrm{R}^{2}\right.$ $=0.979, \mathrm{p}<0.01)$ y DK-7508 $\left(\mathrm{R}^{2}=0.986\right.$, $\mathrm{p}<0.01)$. A través del valor constante $\mathrm{C} 2$ del modelo, se evidenció que la biomasa vegetativa total varió aproximadamente entre
940 y $1.100 \mathrm{~g} \mathrm{MS} \mathrm{m}^{-2}(\bar{X}=1.023$, rango $=$ 162). Los híbridos UTEQ-101 (ver Figura I: a) e INIAP-H551 (ver Figura I: b), acumularon la mitad de la biomasa total aproximadamente a los 26 días (valor constante C3) y el UTEQ101 a los 32 días. La curva de acumulación de biomasa observada fue semejante entre los híbridos UTEQ-101 e INIAP-H551, y mucho más pronunciada que la observada para el híbrido DK-7508, lo que es consistente con los valores constante $\mathrm{C} 4$ más bajos, observados en los híbridos UTEQ-101 $(2,38)$ e INIAP-H551 $(2,38)$ con respecto al observado en el DK$7508(5,65)$.

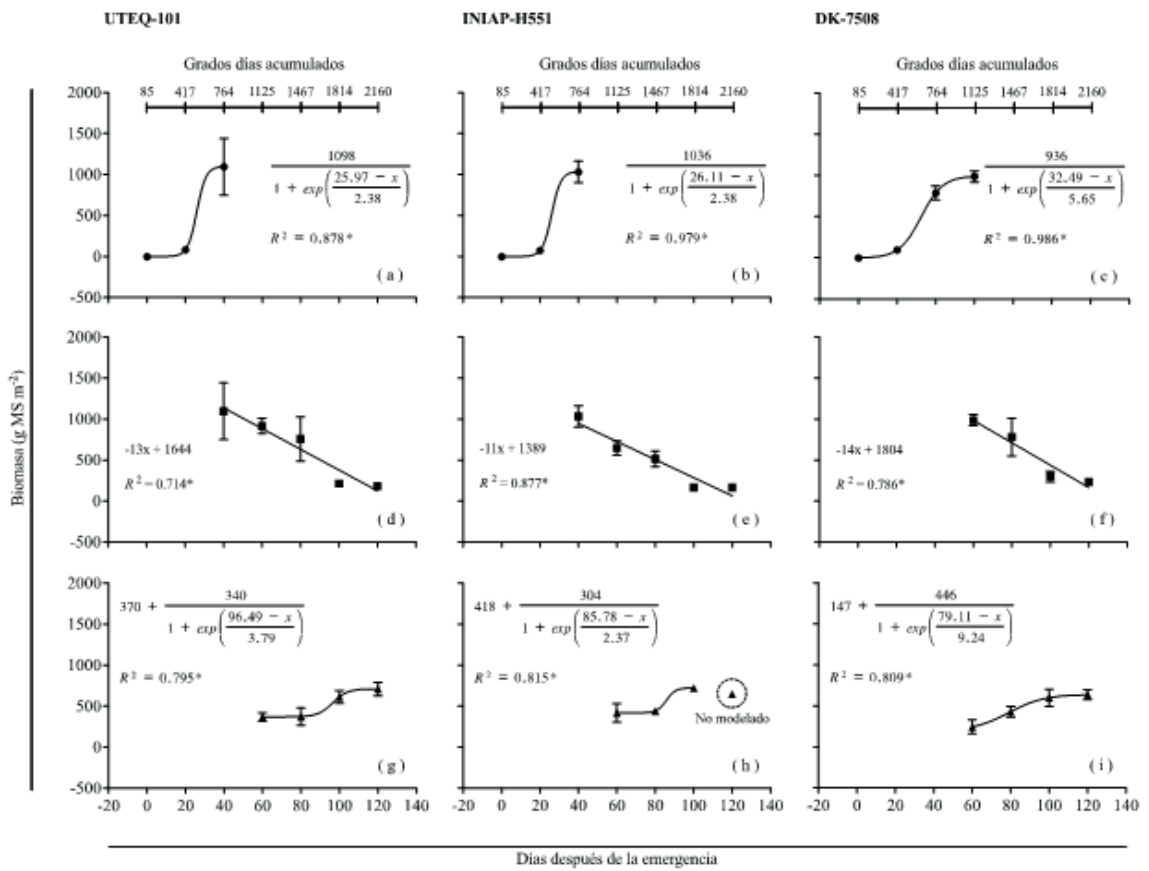

Fuente: Elaboración propia, 2021.

Figura I: Dinámica de acumulación $(=)$ y desacumulación $(<)$ de biomasa vegetativa total y acumulación de biomasa reproductiva ( ) durante el ciclo de cultivo de los híbridos UTEQ-101, INIAP-H551 y DK-7508, cultivados en la zona central del litoral ecuatoriano 
La acumulación de biomasa vegetativa (tallo + hojas) y reproductiva (mazorca), es modelada por la ecuación: $C 1+\frac{C 2}{1+\exp \left(\frac{C_{8-X}}{C_{4}}\right)}$,

donde $C 1, C 2, C 3$ y $C 4$ son valores constantes. Las Figuras representan el promedio y la desviación estándar $(\mathrm{n}=7)$.

La desacumulación de biomasa vegetativa total (ver Figura I: d, e y f) a través del tiempo, mantuvo una tendencia lineal simple, en los híbridos UTEQ-101 $\left(\mathrm{R}^{2}=0.714\right.$, $\mathrm{p}<0.01)$, INIAP-H551 $\left(\mathrm{R}^{2}=0.877, \mathrm{p}<0.01\right)$ y DK-7508 $\left(\mathrm{R}^{2}=0.786, \mathrm{p}<0.01\right)$ con una desacumulación diaria (pendiente de la recta) de 13,11 y $14 \mathrm{~g} \mathrm{MS} \mathrm{m}^{-2}$, respectivamente. En los híbridos UTEQ-101 e INIAP-H551, la desacumulación de biomasa desde los órganos vegetativos se inició a partir de los 40 días, mientras que en el híbrido DK-7508, se inició a partir de los 60 días. La desacumulación de biomasa desde los órganos vegetativos, parece cesar a los 100 días luego de la emergencia de las plántulas de maíz, independientemente del híbrido.

La acumulación de biomasa a nivel de la mazorca (ver Figura I: g, h y i) a través del tiempo, se ajustó a una curva sigmoidea (ecuación de Boltzmann) en los híbridos UTEQ-101 $\left(\mathrm{R}^{2}=0.878, \mathrm{p}<0.01\right)$, INIAP-H551 $\left(\mathrm{R}^{2}=0.979, \mathrm{p}<0.01\right)$ y DK-7508 $\left(\mathrm{R}^{2}=0.986\right.$, $\mathrm{p}<0.01)$. A través del valor constante $\mathrm{C} 1 \mathrm{del}$ modelo, se evidenció que a los 60 días los híbridos UTEQ-101, INIAP-H551 y DK-7508 habían acumulado 370, 418 y $147 \mathrm{~g} \mathrm{MS} \mathrm{m}^{-2}$, respectivamente; y desde los 60 a los 120 días se acumularon 340, 304 y $446 \mathrm{~g} \mathrm{MS} \mathrm{m}^{-2}$ (valor constante $\mathrm{C} 2$ ), respectivamente. Es decir, que los híbridos UTEQ-101, INIAP-H551 y DK-7508 alcanzaron una acumulación total a nivel de mazorca de 710, 722 y $593 \mathrm{~g} \mathrm{MS} \mathrm{m}^{-2}$, respectivamente.

La mitad de la biomasa acumulada entre 60 y 120 días (valor constante C3), se alcanzó entre los 79 (DK-7508) y 96 (UTEQ-101) días. La curva de acumulación de biomasa a nivel de la mazorca, tuvo una pronunciación alta $(2,37)$, media $(3,79)$ y baja $(9,24)$ en los híbridos INIAP-H551, UTEQ-101 y DK7508, respectivamente.

En la Tabla 3, se presenta la absorción y acumulación de nutrientes de los híbridos evaluados de maíz, la misma que fue determinada a los 120 días (2.160 ${ }^{\circ}$ días) luego de la emergencia, en donde se observó que no existe diferencias $(\mathrm{p}>0.05)$ en la absorción total de $\mathrm{P}$ y $\mathrm{S}$ con una absorción media de 3,5 y $1,2 \mathrm{~g} \mathrm{~kg}^{-1}$, respectivamente, lo cual, fue más condicionado por la acumulación a nivel de mazorca, y no por la observada a nivel de tallo y hojas. En cuanto a la absorción de N, K, Ca y $\mathrm{Mg}$, sí se evidenciaron diferencias $(\mathrm{p}<0.05)$. Entre los híbridos UTEQ-101 y DK-7508, no hubo diferencias en la absorción de $\mathrm{N}, \mathrm{K}$ y Ca, con una media de absorción total de 14,3, 11,6 y $11,5 \mathrm{~g} \mathrm{~kg}^{-1}$, respectivamente, mientras que la mayor absorción de $\mathrm{Mg}$ la registró el híbrido UTEQ-101 con 3,3 $\mathrm{g} \mathrm{kg}^{-1}$. En el híbrido INIAP-H551, siempre se observó la menor absorción de N, K, Ca y Mg, con 13,5; 11,0; 9,$9 ;$ y $2,9 \mathrm{~g} \mathrm{k}^{-1}$, respectivamente. 
Tabla 3

Absorción y acumulación de nutrientes primarios ( $N$, P y K) y secundarios (Ca, Mg y S) en tallo, hojas y mazorca en híbridos de maíz cultivados en la zona central del litoral ecuatoriano

\begin{tabular}{|c|c|c|c|c|c|c|c|c|c|}
\hline Órgano & & $\begin{array}{c}\text { UTEQ } \\
101\end{array}$ & & $\begin{array}{c}\text { INIAP } \\
\text { H551 }\end{array}$ & & $\begin{array}{c}\text { DK } \\
7508\end{array}$ & & $\bar{X}$ & \\
\hline \multirow{5}{*}{ Tallo } & $\mathrm{N}$ & 11,98 & $\mathrm{a}$ & 10,18 & $\mathrm{~b}$ & 9,73 & $\mathrm{~b}$ & 10,60 & 0,27 \\
\hline & $\mathrm{P}$ & 1,24 & $\mathrm{a}$ & 0,94 & b & 0,99 & $\mathrm{~b}$ & 1,06 & 0,02 \\
\hline & $\mathrm{K}$ & 15,44 & b & 16,66 & $\mathrm{a}$ & 15,27 & $\mathrm{~b}$ & 15,79 & 0,32 \\
\hline & $\mathrm{Mg}$ & 2,52 & b & 2,72 & $\mathrm{a}$ & 2,83 & $\mathrm{a}$ & 2,69 & 0,05 \\
\hline & $\mathrm{S}$ & 1,34 & $\mathrm{a}$ & 0,61 & $\mathrm{c}$ & 1,19 & $\mathrm{~b}$ & 1,05 & 0,02 \\
\hline \multirow{5}{*}{ Hojas } & $\mathrm{N}$ & 15,82 & $\mathrm{~b}$ & 17,02 & $\mathrm{a}$ & 16,64 & $\mathrm{ab}$ & 16,49 & 0,24 \\
\hline & $\mathrm{P}$ & 1,28 & b & 1,48 & $\mathrm{a}$ & 1,10 & $\mathrm{c}$ & 1,29 & 0,03 \\
\hline & $\mathrm{K}$ & 7,69 & b & 7,08 & $b$ & 9,20 & $\mathrm{a}$ & 7,99 & 0,24 \\
\hline & $\mathrm{Mg}$ & 3,03 & $\mathrm{a}$ & 2,85 & $\mathrm{a}$ & 2,83 & $\mathrm{a}$ & 2,90 & 0,06 \\
\hline & $\mathrm{S}$ & 1,48 & $\mathrm{a}$ & 0,96 & $\mathrm{~b}$ & 0,70 & $\mathrm{c}$ & 1,05 & 0,02 \\
\hline \multirow{5}{*}{ Mazorca } & $\mathrm{N}$ & 14,25 & b & 13,64 & $\mathrm{~b}$ & 15,33 & $\mathrm{a}$ & 14,41 & 0,28 \\
\hline & $\mathrm{P}$ & 4,23 & $\mathrm{a}$ & 4,22 & $\mathrm{a}$ & 4,03 & $\mathrm{a}$ & 4,16 & 0,12 \\
\hline & $\mathrm{K}$ & 11,54 & $\mathrm{a}$ & 10,56 & $\mathrm{~b}$ & 10,95 & $\mathrm{ab}$ & 11,02 & 0,22 \\
\hline & $\mathrm{Mg}$ & 3,48 & $\mathrm{a}$ & 2,92 & $\mathrm{~b}$ & 2,96 & $\mathrm{~b}$ & 3,12 & 0,04 \\
\hline & $\mathrm{S}$ & 1,24 & $\mathrm{a}$ & 1,30 & $\mathrm{a}$ & 1,24 & $\mathrm{a}$ & 1.26 & 0,04 \\
\hline \multirow{5}{*}{ Total } & $\mathrm{N}$ & 14,07 & $a b$ & 13,49 & $\mathrm{~b}$ & 14,57 & $a^{*}$ & 14,04 & 0,23 \\
\hline & $\mathrm{P}$ & 3,62 & $\mathrm{a}$ & 3,59 & $\mathrm{a}$ & 3,22 & $\mathrm{a}$ & 3,48 & 0,11 \\
\hline & $\mathrm{K}$ & 11,78 & $\mathrm{a}$ & 11,02 & $\mathrm{~b}$ & 11,47 & $\mathrm{ab}$ & 11,42 & 0,18 \\
\hline & $\mathrm{Mg}$ & 3,33 & a & 2,89 & $\mathrm{~b}$ & 2,93 & $\mathrm{~b}$ & 3,05 & 0,04 \\
\hline & S & 1,27 & a & 1,19 & $\mathrm{a}$ & 1,18 & $\mathrm{a}$ & 1,21 & 0,03 \\
\hline
\end{tabular}

Nota: * promedios horizontales con letras iguales no son estadísticamente diferentes de acuerdo a la prueba de Tukey al nivel 0.05. EEM, error estándar de la media.

Fuente: Elaboración propia, 2021.

En el presente estudio, tal como era esperado, la biomasa total se distribuye en distintas proporciones en cada uno de los órganos constitutivos de la planta de maíz. Sin embargo, el híbrido de maíz y el tiempo (fenología) transcurrido desde la emergencia (punto de referencia para el inicio de este estudio), condicionan la dinámica de acumulación de biomasa (Koca y Erekul, 2016).

Así, señalando la existencia de variedades e híbridos de maíz con rendimientos muy variables, ya sea asociado al genotipo
(Duvick, 2005) o a las condiciones del medio en donde se desarrolla (Setimela, et al., 2017), se indica que los híbridos evaluados alcanzaron una biomasa total bastante semejante (815 a $891 \mathrm{~g} \mathrm{MS} \mathrm{m}^{-2}$ ), lo que en una buena medida responde a que de manera sistemática se ha venido seleccionando un tipo ideal de panta de maíz (Shelton y Tracy, 2015), es decir, plantas con un tamaño adecuado para evitar el volcamiento y con alturas de inserción de mazorca que facilite la mecanización de la cosecha.

Las diferencias en el patrón de 
distribución de biomasa, indica que un determinado híbrido tiene la posibilidad de ser utilizado dominantemente para la producción forrajera (biomasa vegetativa) o para la producción de semillas (biomasa reproductiva), como fue el caso del DK-7508 y el UTEQ-101, respectivamente, por citar como ejemplos los dos híbridos extremos. Aunque es claro que estrategias agronómicas, como el incremento de plantas por unidad de superficie (Sangoi, 2000; Sadeghi, 2013) o el tratamiento con fitohormonas (Iqbal, et al., 2017), pueden favorecer un determinado tipo de crecimiento (vegetativo, reproductivo).

Por otra parte, tal como ha sido reportado por otros investigadores como Martins et al. (2017), la acumulación de biomasa de los híbridos evaluados fue descrita por una misma función de crecimiento a través del tiempo, destacándose que en el transcurso de los primeros 20 días luego de la emergencia, las hojas son el órgano con mayor prioridad de crecimiento con respecto al tallo, y posteriormente, antes de expresarse el crecimiento reproductivo, la prioridad se invierte (el tallo pasa a ser el órgano prioritario). Al iniciarse el crecimiento reproductivo, aunque la relación tallo/hoja se mantiene sobre uno, la mazorca (brácteas, tuza y granos) pasa a ser el órgano prioritario.

En especies de crecimiento determinado como el trigo, se reconoce una etapa de crecimiento vegetativo y otra de crecimiento reproductivo. La primera, se caracteriza por la acumulación de biomasa a nivel de órganos vegetativos (ej. macollos, hojas); y la segunda, caracterizada por una disminución de la biomasa desde los órganos vegetativos y una acumulación de biomasa a nivel de los órganos reproductivos (ej. espigas) (Singh, et al., 2017).

Las características de crecimiento en las etapas descritas, han sido tomadas como evidencia de la existencia de una removilización de recursos desde los órganos vegetativos hacia los órganos reproductivos (Gao, et al., 2017), lo cual también se refleja en la fuerte relación que existe entre la biomasa total y el rendimiento (índice de cosecha)
(Echarte y Andrade, 2003). Sin embargo, se conoce poco sobre la dinámica (acumulación/ desacumulación) de la biomasa en los órganos de una planta de maíz (tallo, hojas), por lo que estudios como el presente son relevantes al evidenciar que el tallo y las hojas son órganos que, dependiendo del híbrido de maíz, pueden alcanzar su máxima acumulación en etapas tempranas o avanzadas del crecimiento vegetativo.

A nivel del tallo, la acumulación total entre los híbridos fue bastante semejante (572 a $619 \mathrm{~g} \mathrm{MS} \mathrm{m}^{-2}$ ), pero la tasa promedio de acumulación resultó variable (9 a $17 \mathrm{~g}$ MS $\mathrm{m}^{-2}$ día $\left.^{-1}\right)$, y, por lo tanto, determinante de la amplitud del ciclo de acumulación. Algo similar sucedió a nivel de las hojas, la acumulación fue semejante entre híbridos (383 y $420 \mathrm{~g} \mathrm{MS} \mathrm{m}^{-2}$ ) y la tasa de acumulación fue variable (6 a $10 \mathrm{~g} \mathrm{MS} \mathrm{m}^{-2}$ día $\left.^{-1}\right)$. En ambos órganos, tallo y hojas, la desacumulación (tasas con valores negativos) de biomasa, coincidió con la etapa de crecimiento reproductivo, a una tasa relativamente semejante entre híbridos, tanto en tallo (-6 a $-7 \mathrm{~g} \mathrm{MS} \mathrm{m}^{-2}$ día $\left.{ }^{1}\right)$ como para hojas $\left(-5\right.$ a -6 g MS m ${ }^{-2}$ día $\left.^{-1}\right)$, encontrándose que las mayores diferencias entre los híbridos se encuentran determinadas por la amplitud del tiempo de desacumulación, observado tanto en tallo (desde los 60 a los 120 días) como en hojas (desde los 60 a los 100 días).

El conocimiento de la dinámica de la biomasa, que se da en cada uno de los órganos de una planta de maíz, permite establecer la eficiencia de acumulación de recursos y consecuentemente, la contribución de recursos por órgano para la generación de la cosecha, lo que en el campo de ecofisiología y nutrición de cultivos, se conoce como eficiencia de utilización. A partir de lo descrito, es posible identificar nuevos objetivos del fitomejoramiento. Sabiendo que la tasa de desacumulación desde el tallo y desde las hojas, es semejante, y que la amplitud del tiempo de desacumulación es mayor en el tallo, un escenario podría ser seleccionar cultivares de maíz con una alta capacidad de acumulación de biomasa a nivel del tallo. 
Producción de biomasa en cultivos de maiz: Zona central de la costa de Ecuador

Vásconez Montúfar, Gregorio Humberto; Caicedo Acosta, Luis Alberto;

Véliz Zamora, Diana Verónica y Sánchez Mora, Fernando David

El mejoramiento genético a partir de características distintas al rendimiento o la tolerancia a enfermedades, no es una aproximación completamente nueva. En la actualidad, también se observa el interés en características tales como la alta densidad de raíces (Páez-García, et al., 2015), la filotaxia, la eficiencia de utilización de nutrientes o de la radiación.

Para establecer la demanda de nutrientes, que requiere un cultivo para expresar el rendimiento alcanzable en un determinado agroecosistema, se requiere determinar la concentración de nutrientes en el tejido vegetal al final del ciclo vegetativo (Westermann, 2005; Osvalde, 2011), lo que permite estimar el requerimiento de nutrientes para la formación de tallos, hojas y granos, e incluso llegar a estimar la fracción de nutrientes que se recicla en el suelo por la incorporación de los residuos vegetales (Lupwayi, et al., 2005; Abbasi, et al., 2015) y la fracción de nutrientes que se exporta en la cosecha (Ferreira, et al., 2012).

Sobre esta base, en este estudio se determinó la concentración de nutrientes primarios $(\mathrm{N}, \mathrm{P}, \mathrm{K})$ y secundarios $(\mathrm{Mg}, \mathrm{S})$ a nivel de tallos, hojas y granos, a los 120 días (2.160 ${ }^{\circ}$ días) luego de la emergencia, observándose que en términos promedio el principal destino del N, P y K son las hojas (16,6 $\left.\mathrm{g} \mathrm{kg}^{-1}\right)$, las mazorcas $\left(4,2 \mathrm{~g} \mathrm{~kg}^{-1}\right)$ y los tallos $\left(15,8 \mathrm{~g} \mathrm{~kg}^{-1}\right)$, respectivamente, y el principal destino del $\mathrm{Mg}$ y $\mathrm{S}$ fue las mazorcas $\left(\mathrm{Mg}=3,1\right.$ y $\left.\mathrm{S}=1,3 \mathrm{~g} \mathrm{~kg}^{-1}\right)$. Sin embargo, en los estudios de acumulación y particionamiento de nutrientes, se debe considerar que la constante generación de nuevos híbridos y la mejora de las prácticas agrícolas pueden condicionar la absorción, la acumulación y por ende el particionamiento (Bender, et al., 2013).

\section{Conclusiones}

El rendimiento (granos) de los modernos híbridos de maíz evaluados es mayor que el observado en el híbrido antiguo, lo que aparentemente responde dominantemente a una mayor absorción de $\mathrm{N}, \mathrm{K}, \mathrm{Ca}$ y $\mathrm{Mg}$ y no a la acumulación y distribución de biomasa, lo que sugiere que la selección de materiales considerando la proporción de distribución de biomasa, podría contribuir a incrementar la producción de follaje (biomasa vegetativa) o granos (biomasa reproductiva), dependiendo del interés agronómico. Por otra parte, la acumulación de biomasa vegetativa y reproductiva, responde a un modelo sigmoideo y la removilización de biomasa vegetativa para la generación del rendimiento, responde a un modelo lineal.

La diferencia en la cantidad absorbida de $\mathrm{N}$ y K, fue condicionada por la acumulación a nivel de tallo, hojas y mazorcas, mientras que la absorción de $\mathrm{Ca}$, estuvo condicionada únicamente por la acumulación en mazorcas, y la absorción de $\mathrm{Mg}$, por la acumulación en tallo y en mazorcas. Además de lo anterior, se evidencia que por cada kilogramo de biomasa seca total ( $\sin$ considerar raíces) se requiere aproximadamente 14,$0 ; 3,5 ; 11,4 ; 11,3 ; 3,0 ; \mathrm{y}$ 1;2 g de N, P, K, Ca, Mg y S, respectivamente. Finalmente, las materias primas de base lignocelulósica, como el maíz, permiten optimar los sistemas sostenibles para el desarrollo del país, tanto en la cosecha como en el procesamiento de los recursos de biomasas; además, mejorar la eficacia y el rendimiento de los procesos, de este tipo de producto agrícola, con la selección de materiales con mayor capacidad de acumulación/desacumulación, podría contribuir a incrementar el rendimiento de los productores de maíz, un mayor crecimiento y participación en los mercados, y por ende mejor calidad de vida.

\section{Referencias bibliográficas}

Abbasi, M. K., Tahir, M. M., Sabir, N., y Khurshid, M. (2015). Impact of the addition of different plant residues on nitrogen mineralizationimmobilization turnover and carbon content of a soil incubated under laboratory conditions. Solid Earth, 6(1), 197-205. https://doi.org/10.5194/ 
se-6-197-2015

Andrade, F. H., Cirilo, A. G., Uhart, S. A., y Otegui, M.E. (1996). Ecofisiología del cultivo de maíz. Editorial La Barrosa.

Bender, R. R., Haegele, J. W., Ruffo, M. L., y Below, F. E. (2013). Nutrient uptake, partitioning, and remobilization in modern, transgenic insect-protected maize hybrids. Agronomy Journal, 105(1), 161-170. https://doi. org/10.2134/agronj2012.0352

Caicedo, J. C., Puyol, J. L., López, M. C., y Ibáñez, S. S. (2020). Adaptabilidad en el sistema de producción agrícola: Una mirada desde los productos alternativos sostenibles. Revista de Ciencias Sociales (Ve), XXVI(4), 308$327 . \quad$ https://doi.org/10.31876/rcs. v26i4.34665

Díaz-Romeu, R., y Hunter, A. (1982). Metodología de muestreo de suelos, análisis químico de suelos y tejido vegetal $y$ de investigaciones en invernadero. Centro Agronómico Tropical de Investigación y Enseñanza.

Duvick, D. N. (2005). The contribution of breeding to yield advances in maize (Zea mays L.). Advances in Agronomy, 86, 83-145. https://doi.org/10.1016/ S0065-2113(05)86002-X

Echarte, L., y Andrade, F. H. (2003). Harvest index stability of Argentinean maize hybrids released between 1965 and 1993. Field Crops Research, 82(1), 1-12. https://doi.org/10.1016/S03784290(02)00232-0

Ferreira, C. F., Vargas, A. C., Prior, S. A., Reissman, C. B., Dos Santos, N. Z., y Gabardo, J. (2012). Influence of corn (Zea mays L.) cultivar development on grain nutrient concentration. International Journal of Agronomy, 2012, 842582. https:// doi.org/10.1155/2012/842582

Fischer, T., Byerlee, D., y Edmeades, G.
(2014). Crop yields and global food security. Will yield increase continue to feed the world? Australian Centre for International Agricultural Research.

Gao, J., Zhao, B., Dong, S., Liu, P., Ren, B., y Zhang, J. (2017). Response of summer maize photosynthate accumulation and distribution to shading stress assessed by using ${ }^{13} \mathrm{CO}_{2}$ stable isotope tracer in the field. Frontiers in Plant Science, 8, 1-12. https://doi.org/10.3389/ fpls.2017.01821

Iqbal, N., Khan, N. A., Ferrante, A., Trivellini, A., Francini, A., y Khan, M. I. R. (2017). Ethylene role in plant growth, development and senescence: interaction with other phytohormones. Frontiers in Plant Science, 8, 1-19. $\quad$ https://doi.org/10.3389/ fpls.2017.00475

Koca, Y. O., y Erekul, O. (2016). Changes of dry matter, biomass and relative growth rate with different phenological stages of corn. Agriculture and Agricultural Sciencie Procedia, 10, 67-75, https:// doi.org/10.1016/j.aaspro.2016.09.015

Lupwayi, N. Z., Clayton, G. W., O’Donovan, J. T., Harker, K. N., Turkington, T. K., y Soon, Y. K. (2006). Potassium release during decomposition of crop residues under conventional and zero tillage. Canadian Journal of Soil Science, 86(3), 473-481. https://doi. org/10.4141/S05-049

Martins, K. V., Dourado-Neto, D., Reichardt, K., Favarin, J. L., Sartori, F. F., Felisberto, G., y Mello, S. C. (2017). Maize dry matter production and macronutrient extraction model as a new approach for fertilizer rate estimation. Annals of the Brazilian Academy of Sciences, 89(S-1), 705716. https://doi.org/10.1590/00013765201720160525

Melendez,J.R., Velásquez-Rivera, J., ElSalous, 
Producción de biomasa en cultivos de maiz: Zona central de la costa de Ecuador

Vásconez Montúfar, Gregorio Humberto; Caicedo Acosta, Luis Alberto;

Véliz Zamora, Diana Verónica y Sánchez Mora, Fernando David_

A., y Peñalver, A. (2021). Gestión para la producción de biocombustibles $2 \mathrm{G}$ : revisión del escenario tecnológico y económico. Revista Venezolana de Gerencia, 26(93), 78-91. https://doi. org/10.52080/rvg93.07

Monteros, A., y Salvador, S. (2014). Rendimiento de maíz duro seco en el Ecuador. Dirección de Análisis y Procesamiento de la Información, Coordinación General del Sistema de Información Nacional, Ministerio de Agricultura y Ganadería.

Nkebiwe, P. M., Weinmann, M., Bar-Tal, A., y Müller, T. (2016). Fertilizer placement to improve crop nutrient acquisition and yield: A review and metaanalysis. Field Crops Research, 196, 389-401. https://doi.org/10.1016/j. fcr.2016.07.018

Osvalde, A. (2011). Optimization of plant mineral nutrition revisited: The roles of plant requirements, nutrient interactions, and soil properties in fertilization management. Environmental and Experimental Biology, 9, 1-8.

Páez-García, A., Motes, C. M., Scheible, W-R., S., Chen, R., Blancaflor, E. B., y Monteros, M. J. (2015). Root traits and phenotyping strategies for plant improvement. Plants, 4(2), 334-355. https://doi.org/10.3390/plants4020334

Peña, S. E., y López, J. E. (2020). Desarrollo sostenible y oportunidad de aprendizaje de las biorrefinerías: Una alternativa de la biomasa. Revista de Ciencias Sociales (Ve), XXVI(E-2), 401-413. https://doi.org/10.31876/rcs. $\underline{\mathrm{v} 26 \mathrm{i} 0.34135}$

Ray, D. K., Mueller, N. D., West, P. C., y Foley, J. A. (2013). Yield Trends Are Insufficient to Double Global Crop Production by 2050. PLOS ONE, 8, e66428. https://doi.org/10.1371/ journal.pone.0066428
Sadeghi, M. (2013). The determination of plant density on dry matter accumulation, grain yield and yield components of four maize hybrids. International Journal of Agriculture and Crop Sciences, 5, 109-114.

Sangoi, L. (2000). Understanding plant density effects on maize growth and development: an important issue to maximize grain yield. Ciència Rural, 31(1), 159-168. https://doi.org/10.1590/S0103$\underline{84782001000100027}$

Setimela, P. S., Magorokosho, C., Lunduka, R., Gasura, E., Makumbi, D., Tarekegne, A., Cairns, J. E., Ndhlela, T., Erenstein, O., y Mwangi, W. (2017). On-farm yield grains with stresstolerant maize in eastern and southern Africa. Agronomy Journal, 109(2), 406-417. $\quad$ https://doi.org/10.2134/ agronj2015.0540

Shelton, A. C., y Tracy, W. F. (2015). Recurrent selection and participatory plant breeding for improvement of two organic open-pollinated sweet corn (Zea mays L.) populations. Sustainability, 7(5), 5139-5152. https://doi.org/10.3390/su7055139

Singh, P. K., Singh, K. K., Gill, K. K., Niwas, R., Singh, R. S., y Sharma, S. (2017). Dry biomass partitioning of growth and development in wheat (Triricum aestivum L.) crop using CERES-wheat in different agro climatic zones of India. Current Science, 113(4), 752766.

Soil Survey Staff (2014). Keys to soil taxonomy. USDA-Natural Resources Conservation Service.

Vasco, A., Saenz, C., Vasco, S., y Vasco, D. (2017). Comportamiento agronómico y evaluación económica de híbridos de maíz cristalino duro (Zea mays L.) en tres zonas agroecológicas del Litoral ecuatoriano. Siembra, 4(1), 66- 
75. https://doi.org/10.29166/siembra. $\underline{\mathrm{v} 4 \mathrm{i} 1.501}$

Vásquez, A. Y., Chávez, C., Herrera, F., y Carreño, F. (2018). Milpa y seguridad alimentaria: El caso de San Pedro El Alto, México. Revista de Ciencias Sociales (Ve), XXIV(2), 24-36.

Vera, D., Comte, E., Guamán, R., y Mora, J. (2020). Calidad del cultivo de maíz: Rendimiento, sanidad y condiciones agroclimáticas. Revista Venezolana de Gerencia, 25(3), 381-390. https://doi. org/10.37960/rvg.v25i3.33376.

Walker, R. J. (2016). Population Growth and its implications for global security. American Journal of Economics and Sociology, 75(4), 980-1004. https:// doi.org/10.1111/ajes.12161

Westermann, D. T. (2005). Nutritional requirements of potatoes. American Journal of Potato Research, 82, 301-307. https://doi.org/10.1007/ $\underline{\mathrm{BF} 02871960}$ 